\title{
ON A GENERALISATION OF THE FUNDAMENTAL MATRIX AND THE SOLUTION OF OPERATOR EQUATIONS
}

\author{
Marianito R. Rodrigo \\ School of Mathematics and Applied Statistics \\ University of Wollongong \\ Wollongong, NSW, AUSTRALIA 2522
}

\begin{abstract}
We consider a broad class of linear operator equations that includes systems of ordinary differential equations, difference equations and fractionalorder ordinary differential equations. This class also includes operator exponentials and powers, as well as eigenvalue problems and Fredholm integral equations. Many problems in engineering and the physical and natural sciences can be described by such operator equations. We generalise the fundamental matrix to a fundamental operator and provide a new explicit method for obtaining an exact series solution to these types of operator equations, together with sufficient conditions for convergence and error bounds. Illustrative examples are also given.
\end{abstract}

AMS Subject Classification: 34A30, 15A16, 34A08, 39A05, 47A75

Key Words: operator equations; ordinary differential equations; difference equations; fractional ordinary differential equations; eigenvalue problems; operator exponentials and powers

\section{Introduction}

Many problems in applied mathematics, especially in engineering and the physical and natural sciences, can be formulated as linear operator equations in some appropriate space. To motivate the research questions in this article, a sampling

Received: December 31, 2019

(C) 2020 Academic Publications 
of such problems is given as follows; more examples are given in Section 3.

\subsection{Ordinary differential equations}

An initial value problem (IVP) associated with a system of linear first-order ordinary differential equations (ODEs) has the form

$$
x^{\prime}(t)=A x(t), \quad x(0)=x_{0},
$$

where $t \geq 0$ typically represents continuous time, $A$ is an $n \times n$ real or complex matrix and $x(t), x_{0} \in \mathbb{R}^{n}$. A system such as (1.1) arises either from modelling or as a result of linearisation when investigating the stability of equilibrium states of a system of nonlinear ODEs $[1,2]$. It is well known that the unique solution of (1.1) can be expressed as

$$
x(t)=\mathrm{e}^{t A} x_{0},
$$

where $\mathrm{e}^{t A}$ is the matrix exponential defined by the series

$$
\mathrm{e}^{t A}=\sum_{j=0}^{\infty} \frac{t^{j}}{j !} A^{j}
$$

and $A^{0}=I$ is the $n \times n$ identity matrix. The matrix exponential is also known as a fundamental matrix.

The calculation of the matrix exponential is not always straightforward as it entails the summation of an infinite series. Putzer [10] gave an elementary method for calculating the matrix exponential that avoids the use of the Jordan canonical form but which requires only the solution of a recursive system of linear first-order ODEs. Putzer's method is described as follows. Let $\lambda_{1}, \lambda_{2}, \ldots, \lambda_{n}$ denote the eigenvalues of $A$. Define the $n \times n$ matrices $P_{0}, P_{1}, \ldots, P_{n}$ by

$$
P_{0}=I, \quad P_{j}=\left(A-\lambda_{j} I\right) P_{j-1}, \quad 1 \leq j \leq n .
$$

Let the functions $y_{1}, y_{2}, \ldots, y_{n}$, each one depending on $t$, satisfy

$$
\begin{aligned}
& y_{1}^{\prime}(t)=\lambda_{1} y_{1}(t), \quad y_{1}(0)=1, \\
& y_{j+1}^{\prime}(t)=\lambda_{j+1} y_{j+1}(t)+y_{j}(t), \quad y_{j+1}(0)=0, \quad 1 \leq j \leq n-1 .
\end{aligned}
$$

Then a finite expansion of the matrix exponential is

$$
\mathrm{e}^{t A}=\sum_{j=0}^{n-1} y_{j+1}(t) P_{j} .
$$




\subsection{Difference equations}

When $m \in \mathbb{N} \cup\{0\}$, then the analogous IVP for a system of linear first-order difference equations can be considered:

$$
x(m+1)=A x(m), \quad x(0)=x_{0},
$$

where $x(m), x_{0} \in \mathbb{R}^{n}$ and $A$ is an $n \times n$ real or complex invertible matrix. Such systems also arise in biological applications when studying discrete-time processes $[3,4]$. The unique solution of $(1.3)$ is

$$
x(m)=A^{m} x_{0},
$$

where $A^{m}$ is the usual matrix power defined recursively by $A^{0}=I$ and $A^{m}=$ $A A^{m-1}$ for $m \geq 1$. Here, $A^{m}$ is a fundamental matrix.

Elaydi and Harris [5] adapted Putzer's method to calculate the matrix power as

$$
A^{m}=\sum_{j=0}^{n-1} y_{j+1}(m) P_{j},
$$

where the $n \times n$ matrices $P_{0}, P_{1}, \ldots, P_{n}$ are defined as in (1.2) and the sequences $y_{1}, y_{2}, \ldots, y_{n}$, each one depending on $m$, satisfy the IVP

$$
\begin{aligned}
& y_{1}(m)=\lambda_{1} y_{1}(m), \quad y_{1}(0)=1, \\
& y_{j+1}(m)=\lambda_{j+1} y_{j+1}(m)+y_{j}(m), \quad y_{j+1}(0)=0, \quad 1 \leq j \leq n-1 .
\end{aligned}
$$

\subsection{Fractional ordinary differential equations}

The author introduced fractional matrix exponentials in [11], where the associated derivative is either a Caputo or a Riemann-Liouville fractional derivative. Let us recall that for a suitable function $f:(0, \infty) \rightarrow \mathbb{R}$ and $\alpha>0$,

$$
{ }_{0} D_{t}^{-\alpha} f(t)=\frac{1}{\Gamma(\alpha)} \int_{0}^{t}(t-u)^{\alpha-1} f(u) \mathrm{d} u
$$

is called the Riemann-Liouville fractional integral of $f$ of order $\alpha$. Let $\lceil\alpha\rceil$ denote the least integer greater than or equal to $\alpha$. Then the Caputo and Riemann-Liouville fractional derivatives of $f$ of order $\alpha$ are

$$
{ }_{0}^{C} D_{t}^{\alpha} f(t)={ }_{0} D_{t}^{-(\lceil\alpha\rceil-\alpha)} D^{\lceil\alpha\rceil} f(t), \quad{ }_{0} D_{t}^{\alpha} f(t)=D^{\lceil\alpha\rceil}{ }_{0} D_{t}^{-(\lceil\alpha\rceil-\alpha)} f(t),
$$


respectively, where $D^{\lceil\alpha\rceil}$ denotes an ordinary derivative. For a discussion of the fractional calculus, see $[7,9]$ for instance and the list of references therein. When the scalar $f(t)$ is replaced by a matrix $\Phi(t)$, then the above definitions can be applied component-wise.

The Caputo fractional matrix exponential was defined as

$$
\exp _{*}(t A ; \alpha)=\sum_{k=0}^{\lceil\alpha\rceil-1} t^{k} A^{k} E_{\alpha, k+1}\left(t^{\alpha} A^{\alpha}\right),
$$

where $E_{\alpha, \beta}$ (with $\alpha, \beta>0$ ) is the two-parameter Mittag-Leffler function. Note that the roles of $j$ and $k$ are reversed here (see [11]). On the other hand, the Riemann-Liouville fractional matrix exponential was defined as

$$
\exp (t A ; \alpha)=\sum_{k=0}^{\lceil\alpha\rceil-1} t^{\alpha-\lceil\alpha\rceil+k} A^{k} E_{\alpha, \alpha-\lceil\alpha\rceil+k+1}\left(t^{\alpha} A^{\alpha}\right) .
$$

It follows that $\exp _{*}(t A ; 1)=\exp (t A ; 1)=\mathrm{e}^{t A}$. The Caputo fractional matrix exponential is the unique matrix function solution of the IVP

$$
{ }_{0}^{C} D_{t}^{\alpha} \Phi(t)=A^{\alpha} \Phi(t), \quad D^{m} \Phi(0+)=A^{m}, \quad 0 \leq m \leq\lceil\alpha\rceil-1,
$$

where $D^{m}$ is the usual differential operator and $A^{\alpha}$ is an appropriately defined arbitrary matrix power [11]. The Riemann-Liouville fractional matrix exponential is the unique matrix function solution of the IVP

$$
{ }_{0} D_{t}^{\alpha} \Phi(t)=A^{\alpha} \Phi(t), \quad D^{m-\lceil\alpha\rceil+\alpha} \Phi(0+)=A^{m}, \quad 0 \leq m \leq\lceil\alpha\rceil-1 .
$$

Note that $D^{m-\lceil\alpha\rceil+\alpha}$ is a Riemann-Liouville fractional integral operator. It can therefore be shown that

$$
x(t)=\exp _{*}(t A ; \alpha) x_{0} \quad \text { and } \quad x(t)=\exp (t A ; \alpha) x_{0}
$$

are the respective unique solutions of the IVPs

$$
{ }_{0}^{C} D_{t}^{\alpha} x(t)=A^{\alpha} x(t), \quad D^{m} x(0+)=A^{m} x_{0}, \quad 0 \leq m \leq\lceil\alpha\rceil-1
$$

and

$$
{ }_{0} D_{t}^{\alpha} x(t)=A^{\alpha} x(t), \quad D^{m-\lceil\alpha\rceil+\alpha} x(0+)=A^{m} x_{0}, \quad 0 \leq m \leq\lceil\alpha\rceil-1 .
$$

Moreover, a procedure analogous to Putzer's method was derived in [11] to calculate these fractional matrix exponentials. Let $\lambda_{1}, \lambda_{2}, \ldots, \lambda_{n}$ be the 
eigenvalues of $A^{\alpha}$, and define the $n \times n$ matrices $P_{0}, P_{1}, \ldots, P_{n}$ as in (1.2) but with $A$ replaced by $A^{\alpha}$. Then it was shown in [11] that $\exp _{*}(t A ; \alpha)$ and $\exp (t A ; \alpha)$ have the form

$$
\sum_{k=0}^{\lceil\alpha\rceil-1} \sum_{j=0}^{n-1} y_{k+1, j+1}(t) A^{k} P_{j}
$$

where the functions $y_{k+1, j+1}$, with $0 \leq k \leq\lceil\alpha\rceil-1$ and $0 \leq j \leq n-1$, each depend on $t$ and respectively satisfy a recursive system of fractional ODEs of either Caputo or Riemann-Liouville type. Clearly, the usual Putzer's method is recovered when $\alpha=1$.

\subsection{Fredholm integral equations}

Let $a, b \in \mathbb{R}(a<b)$ and $\lambda \in \mathbb{C}$. A Fredholm integral equation problem is to find $x=x(t) \in L^{2}[a, b]$ such that

$$
\lambda x(t)=\int_{a}^{b} K(t, u) x(u) \mathrm{d} u, \quad a \leq t \leq b,
$$

where $K$ is some appropriate kernel function. This is a classical problem in applied mathematics. More recently, such equations were shown to arise naturally in the the theory of signal processing (e.g. the spectral concentration problem [13]) and in physics (e.g. the solution of such equations allows for the experimental spectra to be related to various underlying distributions [12]). Later we will show that a method analogous to Putzer's method can be derived to obtain explicit solutions to Fredholm integral equations.

The consideration of the above problems in Sections 1.1-1.4 leads one to conjecture if a generalisation exists for abstract linear operator equations of the form

$$
L x=A x
$$

where $L$ and $A$ are linear operators on some vector space $X$. The operator $L$ can be thought of as "evolution-like", although this is not a strict requirement as we shall see later. Our objectives are (i) to show that indeed such a generalisation exists for a broad class of linear operator equations and (ii) to provide a unified explicit method for obtaining a solution that generalises Putzer's method and the idea of a fundamental matrix. The above examples, and more, turn out to be special cases of our problem. 
The outline of this article is as follows. In Section 2, we give a precise formulation of the linear operator equation to be considered and prove the main result that provides an explicit solution for it (see Theorem 2.1), as well as obtain some important consequences such as sufficient conditions for convergence (see Corollary 2.3) and the number of terms to take in the series to get to within a desired accuracy (see Corollary 2.5). In Section 3, we give applications of our result to (i) operator exponentials with matrix exponentials as a special case, (ii) operator powers with matrix powers as a special case, (iii) linear eigenvalue problems and (iv) Fredholm integral equations. We give a brief discussion and conclusion in Section 4.

\section{Problem formulation and main result}

Let $X$ be a Banach space over a field $\mathbb{F}$, e.g. $\mathbb{F}=\mathbb{R}$ or $\mathbb{F}=\mathbb{C}$. Denote by $B(X)$ the collection of all bounded linear operators on $X$. Then it is well known that $B(X)$ is a Banach algebra. We wish to consider operator equations of the form

$$
L x=A x
$$

for appropriate operators $A$ and $L$. We assume that $A \in B(X)$. To state the assumptions on $L$, we need to introduce some preliminary hypotheses. Let $\left(U_{j}\right)_{j=1}^{\infty}$ be a sequence in $B(X)$ and $\left(c_{j}\right)_{j=1}^{\infty}$ a sequence in $\mathbb{F}$ such that

$$
\sum_{j=1}^{\infty}\left|c_{j+1}\right|\left\|A-U_{j}\right\| \cdots\left\|A-U_{1}\right\|<\infty .
$$

Take $T_{0}=I$ (the identity operator on $X$ ) and define the sequence $\left(T_{j}\right)_{j=1}^{\infty}$ in $B(X)$ by

$$
T_{j}=\left(A-U_{j}\right) T_{j-1}, \quad j \geq 1 .
$$

It follows that

$$
T_{j}=\left(A-U_{j}\right) \cdots\left(A-U_{1}\right), \quad j \geq 1 .
$$

Suppose that $L$ is an an operator (not necessarily bounded) with domain $D(L) \subset$ $X$ such that

$$
L\left(\sum_{j=0}^{\infty} c_{j+1} T_{j} x_{0}\right)=\sum_{j=0}^{\infty} L\left(c_{j+1} T_{j} x_{0}\right)
$$

and

$$
\begin{aligned}
& L\left(c_{1} x_{0}\right)-c_{1} U_{1} x_{0}, \\
& L\left(c_{j+1} T_{j} x_{0}\right)-c_{j+1} U_{j+1} T_{j} x_{0}-c_{j} T_{j} x_{0}, \quad j \geq 1
\end{aligned}
$$


for every $x_{0} \in X$. The goal is to show that $x=T x_{0}$, where $T=\sum_{j=0}^{\infty} c_{j+1} T_{j}$, is an explicit solution of the operator equation (2.1).

Theorem 2.1. Assume that $A \in B(X)$. Suppose that $\left(U_{j}\right)_{j=1}^{\infty}$ is a sequence in $B(X)$ and $\left(c_{j}\right)_{j=1}^{\infty}$ is a sequence in $\mathbb{F}$ such that the convergence condition (2.2) holds. Let $L$ be an operator with domain $D(L) \subset X$ that satisfies (2.4) and (2.5), where the sequence $\left(T_{j}\right)_{j=1}^{\infty}$ in $B(X)$ is given by $(2.3)$. Then

$$
x=T x_{0}, \quad T=\sum_{j=0}^{\infty} c_{j+1} T_{j}
$$

satisfies the operator equation (2.1) for every $x_{0} \in X$.

Remark 2.2. To motivate the hypotheses of Theorem 2.1, it is worthwhile to compare them with those of Putzer's method for calculating the matrix exponential. The sequence $\left(U_{j}\right)_{j=1}^{\infty}$ is the analogue of the eigenvalues $\lambda_{1}, \lambda_{2}, \ldots, \lambda_{n}$ of the $n \times n$ matrix $A$, the sequence $\left(T_{j}\right)_{j=1}^{\infty}$ corresponds to the auxiliary matrices $P_{0}, P_{1}, \ldots, P_{n}$, the sequence $\left(c_{j}\right)_{j=1}^{\infty}$ generalises $y_{1}(t), y_{2}(t), \ldots, y_{n}(t)$ and (2.5) corresponds to their associated ODEs. We can think of $T$ as a "fundamental operator" since it generalises the fundamental matrix $\mathrm{e}^{t A}$.

Proof. If (2.2) holds, then the series $\sum_{j=0}^{\infty} c_{j+1} T_{j}$ converges absolutely. But $B(X)$ is complete, so $\sum_{j=0}^{\infty} c_{j+1} T_{j}$ converges to some $T \in B(X)$, i.e.

$$
T=\sum_{j=0}^{\infty} c_{j+1} T_{j} \quad \text { or } \quad \lim _{n \rightarrow \infty}\left\|T-\sum_{j=0}^{n-1} c_{j+1} T_{j}\right\|=0
$$

We claim that $A x=\sum_{j=0}^{\infty} c_{j+1} A T_{j} x_{0}$. Indeed, if $x=T x_{0}$, then $A x=A\left(T x_{0}\right)=$ $(A T) x_{0}$ and

$$
\begin{aligned}
\left\|(A T) x_{0}-\sum_{j=0}^{n-1} c_{j+1} A T_{j} x_{0}\right\| & =\left\|(A T) x_{0}-\left(A \sum_{j=0}^{n-1} c_{j+1} T_{j}\right) x_{0}\right\| \\
& \leq\|A\|\left\|T-\sum_{j=0}^{n-1} c_{j+1} T_{j}\right\|\left\|x_{0}\right\| .
\end{aligned}
$$

This proves the claim since

$$
\lim _{n \rightarrow \infty}\left\|(A T) x_{0}-\sum_{j=0}^{n-1} c_{j+1} A T_{j} x_{0}\right\|=0 \quad \text { or } \quad A x=\sum_{j=0}^{\infty} c_{j+1} A T_{j} x_{0} .
$$


We see from (2.3) that $T_{j+1}=\left(A-U_{j+1}\right) T_{j}$ for $j \geq 0$; hence

$$
A T_{j}=\left(A-U_{j+1}+U_{j+1}\right) T_{j}=T_{j+1}+U_{j+1} T_{j}
$$

and, combining this with (2.4) and (2.7), we obtain

$$
\begin{aligned}
L x-A x & =\sum_{j=0}^{\infty} L\left(c_{j+1} T_{j} x_{0}\right)-\sum_{j=0}^{\infty} c_{j+1}\left(T_{j+1}+U_{j+1} T_{j}\right) x_{0} \\
& =\sum_{j=0}^{\infty} L\left(c_{j+1} T_{j} x_{0}\right)-\sum_{j=0}^{\infty} c_{j+1} T_{j+1} x_{0}-\sum_{j=0}^{\infty} c_{j+1} U_{j+1} T_{j} x_{0} \\
& =\sum_{j=0}^{\infty} L\left(c_{j+1} T_{j} x_{0}\right)-\sum_{j=1}^{\infty} c_{j} T_{j} x_{0}-\sum_{j=0}^{\infty} c_{j+1} U_{j+1} T_{j} x_{0} .
\end{aligned}
$$

Rearranging terms and using (2.5) yields

$$
\begin{gathered}
L x-A x=L\left(c_{1} x_{0}\right)+\sum_{j=1}^{\infty} L\left(c_{j+1} T_{j} x_{0}\right)-\sum_{j=1}^{\infty} c_{j} T_{j} x_{0} \\
-c_{1} U_{1} x_{0}-\sum_{j=1}^{\infty} c_{j+1} U_{j+1} T_{j} x_{0}=0 .
\end{gathered}
$$

Hence $L x-A x=0$, i.e. $x=T x_{0}$ is an explicit solution of (2.1).

Next we give some sufficient conditions on $\left(U_{j}\right)_{j=1}^{\infty}$ and $\left(c_{j}\right)_{j=1}^{\infty}$ that guarantee the convergence condition (2.2).

Corollary 2.3. With the same assumptions as in Theorem 2.1, suppose that the following conditions hold:

(i) $r \geq \sup _{j \geq 1}\left\|U_{j}\right\|$ and $R>\|A\|+2 r$.

(ii) $c_{j+1}=O\left((R-r)^{-j}\right)$ as $j \rightarrow \infty$.

Then (2.2) holds and therefore (2.6) is a solution of (2.1).

Remark 2.4. Condition (i) states that $\left(U_{j}\right)_{j=1}^{\infty}$ is uniformly bounded by $r$ and that $R>2 r>r$. Condition (ii) assumes that $c_{j+1}$ decays to zero sufficiently fast as $j$ becomes large. 
Proof. From (i) we see that

$$
\left\|A-U_{j}\right\| \leq\|A\|+\left\|U_{j}\right\| \leq\|A\|+r, \quad j \geq 1,
$$

while (ii) implies that there exist $K>0$ and $J \geq 1$ such that

$$
\left|c_{j+1}\right| \leq K(R-r)^{-j}, \quad j \geq J
$$

Define

$$
\rho=\frac{\|A\|+r}{R-r} \in(0,1)
$$

Then

$$
\sum_{j=J}^{\infty}\left|c_{j+1}\right|\left\|A-U_{j}\right\| \cdots\left\|A-U_{1}\right\| \leq K \sum_{j=J}^{\infty} \rho^{j}<\infty
$$

since $0<\rho<1$ in (2.9). Therefore (2.2) holds and the conclusion follows from Theorem 2.1.

The next corollary prescribes the number of terms to take in the series (2.6) to get to within a desired tolerance.

Corollary 2.5. Assume the hypotheses of Corollary 2.3 with $J=1$ in (2.8). Define

$$
N_{\epsilon}=\frac{\log \frac{\epsilon(1-\rho)}{K\left\|x_{0}\right\|}}{\log \rho}, \quad x_{0} \neq 0,
$$

and the error after $n$ terms as

$$
E_{n}=x-\sum_{j=0}^{n-1} c_{j+1} T_{j} x_{0} .
$$

Then for every $\epsilon>0$, we have $\left\|E_{n}\right\|<\epsilon$ whenever $n>N_{\epsilon}$.

Proof. Using (2.8), it is straightforward to see that

$$
\begin{aligned}
\left\|E_{n}\right\| & \leq \sum_{j=n}^{\infty}\left|c_{j+1}\right|\left\|A-U_{j}\right\| \cdots\left\|A-U_{1}\right\|\left\|x_{0}\right\| \\
& \leq K\left\|x_{0}\right\| \sum_{j=n}^{\infty} \rho^{j}=K\left\|x_{0}\right\| \frac{\rho^{n}}{1-\rho}
\end{aligned}
$$

and the result follows after rearranging the expression $n>N_{\epsilon}$. 


\section{Illustrative examples}

In this section, we give several problems to illustrate Theorem 2.1 and Corollary 2.3 .

Example 3.1 (Operator exponential). Suppose that $r>0$ and $R>$ $\|A\|+2 r$. This implies that $R>2 r>r$. Take any sequence $\left(\alpha_{j}\right)_{j=1}^{\infty}$ in $\bar{D}_{r}(0)=\{z \in \mathbb{C}:|z| \leq r\}$. For $t>0$, define

$$
y_{1}(t)=\mathrm{e}^{\alpha_{1} t}, \quad y_{j+1}(t)=\int_{0}^{t} \mathrm{e}^{\alpha_{j+1}(t-u)} y_{j}(u) \mathrm{d} u, \quad j \geq 1 .
$$

It is not difficult to show that

$$
y_{1}^{\prime}(t)=\alpha_{1} y_{1}(t), \quad y_{j+1}^{\prime}(t)=\alpha_{j+1} y_{j+1}(t)+y_{j}(t), \quad j \geq 1 .
$$

Take $U_{j}=\alpha_{j} I$ and $c_{j}=y_{j}(t)$ for each $j \geq 1$ in Theorem 2.1. Then

$$
T(t)=y_{1}(t) I+\sum_{j=1}^{\infty} y_{j+1}(t)\left(A-\alpha_{j} I\right) \cdots\left(A-\alpha_{1} I\right) .
$$

Take $A \in B(X)$. Define

$$
L(x(t))=x^{\prime}(t)=\lim _{h \rightarrow 0} \frac{1}{h}[x(t+h)-x(t)] .
$$

To verify (2.4), we see that

$$
\begin{aligned}
& L\left(\sum_{j=0}^{\infty} y_{j+1}(t) T_{j} x_{0}\right) \\
& \quad=\lim _{h \rightarrow 0} \frac{1}{h}\left[\sum_{j=0}^{\infty} y_{j+1}(t+h) T_{j} x_{0}-\sum_{j=0}^{\infty} y_{j+1}(t) T_{j} x_{0}\right] \\
& \quad=\lim _{h \rightarrow 0} \sum_{j=0}^{\infty} \frac{1}{h}\left[y_{j+1}(t+h)-y_{j+1}(t)\right] T_{j} x_{0} .
\end{aligned}
$$

The next series of lemmas are needed to justify the interchange of the limit and the summation in (3.2), as well as to verify the hypotheses of Corollary 2.3. We note that

$$
\sup _{j \geq 1}\left\|U_{j}\right\|=\sup _{j \geq 1}\left\|\alpha_{j} I\right\|=\sup _{j \geq 1}\left|\alpha_{j}\right| \leq r
$$


thus Corollary 2.3 (i) is true. The following lemma shows that $y_{j+1}(t)=O((R-$ $r)^{-j}$ ) as $j \rightarrow \infty$ and therefore Corollary 2.3 (ii) holds.

Lemma 3.2. For every $t>0$, there holds

$$
\left|y_{j+1}(t)\right| \leq \frac{\mathrm{e}^{R t}}{(R-r)^{j}}, \quad j \geq 1
$$

Proof. As $\operatorname{Re} z \leq|\operatorname{Re} z| \leq|z| \leq r<R$ for $z \in \bar{D}_{r}(0)$, we see from (3.1) that

$$
\left|y_{1}(t)\right|=\mathrm{e}^{\operatorname{Re} \alpha_{1} t} \leq \frac{\mathrm{e}^{R t}}{(R-r)^{0}} .
$$

Now suppose that (3.3) is true for $j$. Then (3.1) again gives

$$
\left|y_{j+2}(t)\right| \leq \int_{0}^{t} \mathrm{e}^{\operatorname{Re} \alpha_{j+1}(t-u)}\left|y_{j+1}(u)\right| \mathrm{d} u \leq \int_{0}^{t} \mathrm{e}^{r(t-u)} \frac{\mathrm{e}^{R u}}{(R-r)^{j}} \mathrm{~d} u .
$$

But

$$
\frac{\mathrm{e}^{r t}}{(R-r)^{j}} \int_{0}^{t} \mathrm{e}^{(R-r) u} \mathrm{~d} u=\frac{\mathrm{e}^{r t}}{(R-r)^{j+1}}\left[\mathrm{e}^{(R-r) t}-1\right] \leq \frac{\mathrm{e}^{R t}}{(R-r)^{j+1}},
$$

which implies that

$$
\left|y_{j+2}(t)\right| \leq \frac{\mathrm{e}^{R t}}{(R-r)^{j+1}}
$$

and thus proves the lemma.

Lemma 3.3. Let $a>0$. Then there exists $h_{0}>0$ (which may depend on a) such that

$$
\frac{\left|\mathrm{e}^{a h}-1\right|}{|h|} \leq 2 a, \quad 0<|h| \leq h_{0} .
$$

In particular,

$$
\frac{\mathrm{e}^{a \bar{h}}-1}{\bar{h}}=\frac{\left|\mathrm{e}^{a \bar{h}}-1\right|}{|\bar{h}|} \leq 2 a, \quad 0<\bar{h} \leq h_{0} .
$$

Proof. We observe that

$$
\frac{\left|\mathrm{e}^{a h}-1\right|}{|h|}=\frac{\mathrm{e}^{a h}-1}{|h|}, \quad h \neq 0 .
$$


Define $g(h)=\mathrm{e}^{a h}-2 a h-1$. Then $g(0)=0$ and $g(\infty)=\infty$. Furthermore, $g$ is convex with a global minimum at $\frac{\log (2)}{a}$ and $g\left(\frac{\log (2)}{a}\right)<0$. It follows that there exists a unique $h_{0}>\frac{\log (2)}{a}>0$ (which may depend on $a$ ) such that $g\left(h_{0}\right)=0$. Comparing the slopes of $h \mapsto \mathrm{e}^{a h}$ and $h \mapsto 2 a h+1$ at $h=0$, we deduce that $\mathrm{e}^{a h} \leq 2 a h+1$ if $0<h \leq h_{0}$ and $2 a h+1<\mathrm{e}^{a h}$ if $h<0$. Combining, we obtain

$$
\frac{\mathrm{e}^{a h}-1}{h} \leq 2 a, \quad 0<|h| \leq h_{0} .
$$

The second result is obvious from the first.

Lemma 3.4. If $z \in \bar{D}_{r}(0)$ and $h \in \mathbb{R} \backslash\{0\}$, then

$$
\frac{\mathrm{e}^{z h}-1}{|h|} \leq \frac{\mathrm{e}^{r|h|}-1}{|h|}
$$

Proof. Since $|z| \leq r$ and $h \neq 0$, the result follows from

$$
\frac{\mathrm{e}^{z h}-1}{h}=\frac{1}{h} \sum_{k=1}^{\infty} \frac{h^{k} z^{k}}{k !} .
$$

Remark 3.5. Using Lemma 3.3, there exists $h_{1}>0$ (which may depend on $R-r$ ) such that

$$
\frac{\left|\mathrm{e}^{(R-r) h}-1\right|}{|h|} \leq 2(R-r), \quad 0<|h| \leq h_{1} .
$$

Moreover, there exists $h_{2}>0$ (which may depend on $r$ ) such that

$$
\frac{\mathrm{e}^{r|h|}-1}{|h|} \leq 2 r, \quad 0<|h| \leq h_{2}
$$

In the following, take $h_{0}=\min \left(h_{1}, h_{2}\right)$ and $K=2\left[r+(R-r) \mathrm{e}^{r h_{0}}\right]$.

Lemma 3.6. Fix $t>0$. For all $j \geq 0$, there holds

$$
\frac{\left|y_{j+1}(t+h)-y_{j+1}(t)\right|}{|h|} \leq \frac{K \mathrm{e}^{R t}}{(R-r)^{j}}, \quad 0<|h| \leq h_{0} .
$$


Proof. Eq. (3.1) yields

$$
\begin{aligned}
y_{j+1}(t+h) & =\int_{0}^{t+h} \mathrm{e}^{\alpha_{j+1}(t+h-u)} y_{j}(u) \mathrm{d} u \\
& =\mathrm{e}^{\alpha_{j+1} h} y_{j+1}(t)+\mathrm{e}^{\alpha_{j+1} h} \int_{t}^{t+h} \mathrm{e}^{\alpha_{j+1}(t-u)} y_{j}(u) \mathrm{d} u
\end{aligned}
$$

from which we deduce that

$$
\begin{aligned}
\frac{y_{j+1}(t+h)-y_{j+1}(t)}{h}= & \frac{\mathrm{e}^{\alpha_{j+1} h}-1}{h} y_{j+1}(t) \\
& +\frac{\mathrm{e}^{\alpha_{j+1} h}}{h} \int_{t}^{t+h} \mathrm{e}^{\alpha_{j+1}(t-u)} y_{j}(u) \mathrm{d} u .
\end{aligned}
$$

This can be rewritten as

$$
\begin{aligned}
& \frac{y_{j+1}(t+h)-y_{j+1}(t)}{h}=\frac{\mathrm{e}^{\alpha_{j+1} h}-1}{h} y_{j+1}(t) \\
& +\frac{\mathrm{e}^{\alpha_{j+1} h}-1}{h} \int_{t}^{t+h} \mathrm{e}^{\alpha_{j+1}(t-u)} y_{j}(u) \mathrm{d} u+\frac{1}{h} \int_{t}^{t+h} \mathrm{e}^{\alpha_{j+1}(t-u)} y_{j}(u) \mathrm{d} u .
\end{aligned}
$$

From Lemma 3.2, we obtain

$$
\left|\int_{t}^{t+h} \mathrm{e}^{\alpha_{j+1}(t-u)} y_{j}(u) \mathrm{d} u\right| \leq \frac{\mathrm{e}^{R t}}{(R-r)^{j}}\left|\mathrm{e}^{(R-r) h}-1\right|, \quad h \neq 0 .
$$

Again using Lemmas 3.2 and 3.4, we see that

$$
\begin{aligned}
& \left|\frac{y_{j+1}(t+h)-y_{j+1}(t)}{h}\right| \leq \frac{\mathrm{e}^{r|h|}-1}{|h|} \frac{\mathrm{e}^{R t}}{(R-r)^{j}} \\
& +\frac{\mathrm{e}^{r|h|}-1}{|h|} \frac{\mathrm{e}^{R t}}{(R-r)^{j}}\left|\mathrm{e}^{(R-r) h}-1\right|+\frac{1}{|h|} \frac{\mathrm{e}^{R t}}{(R-r)^{j}}\left|\mathrm{e}^{(R-r) h}-1\right| \\
& \quad=\frac{\mathrm{e}^{r|h|}-1}{|h|} \frac{\mathrm{e}^{R t}}{(R-r)^{j}}+\frac{\mathrm{e}^{r|h|}}{|h|} \frac{\mathrm{e}^{R t}}{(R-r)^{j}}\left|\mathrm{e}^{(R-r) h}-1\right| .
\end{aligned}
$$

For $0<|h| \leq h_{0}$, Remark 3.5 gives

$$
\left|\frac{y_{j+1}(t+h)-y_{j+1}(t)}{h}\right| \leq 2 r \frac{\mathrm{e}^{R t}}{(R-r)^{j}}+2(R-r) \mathrm{e}^{r h_{0}} \frac{\mathrm{e}^{R t}}{(R-r)^{j}}
$$




$$
=\frac{K \mathrm{e}^{R t}}{(R-r)^{j}} .
$$
that

We are now ready to complete the verification of (2.4) using (3.2). Note

$$
\left\|T_{j}\right\| \leq\left\|A-\alpha_{j} I\right\| \cdots\left\|A-\alpha_{1} I\right\| \leq(\|A\|+r)^{j}, \quad j \geq 1 .
$$

From Lemma 3.6, we have

$$
\begin{aligned}
\left\|\frac{y_{j+1}(t+h)-y_{j+1}(t)}{h} T_{j} x_{0}\right\| & \leq \frac{\left|y_{j+1}(t+h)-y_{j+1}(t)\right|}{|h|}\left\|T_{j}\right\|\left\|x_{0}\right\| \\
& \leq K_{j},
\end{aligned}
$$

where $K_{j}=K \mathrm{e}^{R t}\left\|x_{0}\right\| \rho^{j}$ and $\rho$ is as in (2.9). Since $\sum_{j=0}^{\infty} K_{j}$ is convergent, we conclude that

$$
\sum_{j=0}^{\infty} \frac{y_{j+1}(t+h)-y_{j+1}(t)}{h} T_{j} x_{0}
$$

converges uniformly for $0<|h| \leq h_{0}$. Thus (3.2) gives

$$
\begin{aligned}
L\left(\sum_{j=0}^{\infty} y_{j+1}(t) T_{j} x_{0}\right) & =\sum_{j=0}^{\infty} \lim _{h \rightarrow 0} \frac{y_{j+1}(t+h)-y_{j+1}(t)}{h} T_{j} x_{0} \\
& =\sum_{j=0}^{\infty} y_{j+1}^{\prime}(t) T_{j} x_{0}=\sum_{j=0}^{\infty} L\left(y_{j+1}(t) T_{j} x_{0}\right)
\end{aligned}
$$

and this completes the verification of (2.4).

The last thing we need to check is (2.5). We see that

$$
\begin{aligned}
& L\left(c_{1} x_{0}\right)-c_{1} U_{1} x_{0}=\left[y_{1}^{\prime}(t)-\alpha_{1} y_{1}(t)\right] x_{0}=0, \\
& L\left(c_{j+1} T_{j} x_{0}\right)-c_{j+1} U_{j+1} T_{j} x_{0}-c_{j} T_{j} x_{0} \\
& \quad=\left[y_{j+1}^{\prime}(t)-\alpha_{j+1} y_{j+1}(t)-y_{j}(t)\right] T_{j} x_{0}=0, \quad j \geq 1 .
\end{aligned}
$$

Since both conditions (i) and (ii) of Corollary 2.3 have been verified, (2.2) holds and a solution of $x^{\prime}(t)=A x(t)$ is given by

$$
\begin{aligned}
x(t) & =T(t) x_{0}, \\
T(t) & =y_{1}(t) I+\sum_{j=1}^{\infty} y_{j+1}(t)\left(A-\alpha_{j} I\right) \cdots\left(A-\alpha_{1} I\right) .
\end{aligned}
$$


As $R$ can be taken arbitrarily large as long as $R>\|A\|+2 r$, (3.4) is valid for any sequence $\left(\alpha_{j}\right)_{j=1}^{\infty}$ in $\mathbb{C}$ such that $\left|\alpha_{j}\right| \leq r$ for $j \geq 1$.

The simplest case to choose $\left(\alpha_{j}\right)_{j=1}^{\infty}=(0,0, \ldots)$. Eq. (3.1) gives $y_{j}(t)=$ $\frac{t^{j-1}}{(j-1) !}$ for all $j \geq 1$ and (3.4) simplifies to

$$
x(t)=T(t) x_{0}, \quad T(t)=I+\sum_{j=1}^{\infty} \frac{t^{j}}{j !} A^{j}=\exp (t A),
$$

thus recovering the operator exponential.

Another choice, for example, is $\left(\alpha_{j}\right)_{j=1}^{\infty}=(\alpha, 0, \ldots)$, where $\alpha \in \mathbb{C} \backslash\{0\}$. We see from (3.1) that $y_{1}(t)=\mathrm{e}^{\alpha t}$, and claim that

$$
y_{j+1}(t)=\frac{1}{\alpha^{j}}\left[\mathrm{e}^{\alpha t}-\sum_{k=0}^{j-1} \frac{(\alpha t)^{k}}{k !}\right], \quad j \geq 1 .
$$

Eq. (3.1) gives

$$
y_{2}(t)=\int_{0}^{t} \mathrm{e}^{\alpha u} \mathrm{~d} u=\frac{1}{\alpha}\left(\mathrm{e}^{\alpha t}-1\right) .
$$

Suppose that the claim is true for $j$. Then

$$
\begin{aligned}
y_{j+2}(t) & =\int_{0}^{t} y_{j+1}(u) \mathrm{d} u=\frac{1}{\alpha^{j}} \int_{0}^{t}\left[\mathrm{e}^{\alpha u}-\sum_{k=0}^{j-1} \frac{(\alpha u)^{k}}{k !}\right] \mathrm{d} u \\
& =\frac{1}{\alpha^{j+1}}\left(\mathrm{e}^{\alpha t}-1\right)-\frac{1}{\alpha^{j}} \sum_{k=0}^{j-1} \frac{\alpha^{k}}{k !} \frac{t^{k+1}}{k+1} .
\end{aligned}
$$

But

and therefore

$$
\sum_{k=0}^{j-1} \frac{\alpha^{k}}{k !} \frac{t^{k+1}}{k+1}=\frac{1}{\alpha} \sum_{k=0}^{j-1} \frac{(\alpha t)^{k+1}}{(k+1) !}=\frac{1}{\alpha} \sum_{k=1}^{j} \frac{(\alpha t)^{k}}{k !}
$$

$$
y_{j+2}(t)=\frac{1}{\alpha^{j+1}}\left(\mathrm{e}^{\alpha t}-1\right)-\frac{1}{\alpha^{j+1}} \sum_{k=1}^{j} \frac{(\alpha t)^{k}}{k !}=\frac{1}{\alpha^{j+1}}\left[\mathrm{e}^{\alpha t}-\sum_{k=0}^{j} \frac{(\alpha t)^{k}}{k !}\right],
$$

which proves the claim. Hence (3.4) yields $x(t)=T(t) x_{0}$, where

$$
T(t)=\mathrm{e}^{\alpha t} I+\sum_{j=1}^{\infty} \frac{1}{\alpha^{j}}\left[\mathrm{e}^{\alpha t}-\sum_{k=0}^{j-1} \frac{(\alpha t)^{k}}{k !}\right] A^{j-1}(A-\alpha I) .
$$


Remark 3.7. If we impose the initial condition $x(0)=x_{0}$ on $x^{\prime}(t)=$ $A x(t)$, then the unique solution of this IVP is

$$
x(t)=\exp (t A) x_{0} .
$$

Uniqueness of the solution yields

$$
\exp (t A)=\mathrm{e}^{\alpha t} I+\sum_{j=1}^{\infty} \frac{1}{\alpha^{j}}\left[\mathrm{e}^{\alpha t}-\sum_{k=0}^{j-1} \frac{(\alpha t)^{k}}{k !}\right] A^{j-1}(A-\alpha I)
$$

as a non-power series representation of the operator exponential. More generally,

$$
\exp (t A)=y_{1}(t) I+\sum_{j=1}^{\infty} y_{j+1}(t)\left(A-\alpha_{j} I\right) \cdots\left(A-\alpha_{1} I\right)
$$

for any uniformly bounded sequence $\left(\alpha_{j}\right)_{j=1}^{\infty}$ in $\mathbb{C}$.

Example 3.8 (Putzer method for the matrix exponential). Continuing with the previous example, we now consider whether the sequence $\left(\alpha_{j}\right)_{j=1}^{\infty}$ can be chosen in such a way that the series in (3.4) collapses to a finite sum. This can be done if $X$ is finite dimensional, say $X=\mathbb{R}^{n}$, due to the Cayley-Hamilton Theorem.

Let $x(t), x_{0} \in \mathbb{R}^{n}$. Suppose that $A \in M_{n}(\mathbb{R})$ has eigenvalues $\lambda_{1}, \lambda_{2}, \ldots, \lambda_{n}$. Then $x^{\prime}(t)=A x(t)$ simplifies to a system of $n$ linear first-order ODEs. Choose $\alpha_{j}=\lambda_{j}$ for $1 \leq j \leq n$ and $\alpha_{j}=0$ for $j \geq n+1$. The Cayley-Hamilton Theorem implies that

$$
\left(A-\alpha_{j} I\right) \cdots\left(A-\alpha_{1} I\right)=O, \quad j \geq n,
$$

where $O$ is the $n \times n$ zero matrix. Therefore we have $x(t)=T(t) x_{0}$, where

$$
T(t)=y_{1}(t) I+\sum_{j=1}^{n-1} y_{j+1}(t)\left(A-\lambda_{j} I\right) \cdots\left(A-\lambda_{1} I\right)
$$

and

$$
y_{1}(t)=\mathrm{e}^{\lambda_{1} t}, \quad y_{j+1}(t)=\int_{0}^{t} \mathrm{e}^{\lambda_{j+1}(t-u)} y_{j}(u) \mathrm{d} u, \quad 1 \leq j \leq n-1 .
$$

Given the initial condition $x(0)=x_{0}$, then uniqueness of the solution yields

$$
\exp (t A)=y_{1}(t) I+\sum_{j=1}^{n-1} y_{j+1}(t)\left(A-\lambda_{j} I\right) \cdots\left(A-\lambda_{1} I\right)
$$

and we recover the Putzer method for computing the matrix exponential [10]. 
Example 3.9 (Operator power). Let $r>0$ and $R>\|A\|+2 r$. Then $R>2 r>r$. Take any sequence $\left(\alpha_{j}\right)_{j=1}^{\infty}$ belonging to $\bar{D}_{r}(0)=\{z \in \mathbb{C}:|z| \leq r\}$. For any $m \in \mathbb{N} \cup\{0\}$, define

$$
y_{1}(m)=\alpha_{1}^{m}, \quad y_{j+1}(m)=\sum_{k=0}^{m-1} \alpha_{j+1}^{m-1-k} y_{j}(k), \quad j \geq 1 .
$$

It is not difficult to show that

$$
\begin{aligned}
y_{1}(m+1) & =\alpha_{1} y_{1}(m), \\
y_{j+1}(m+1) & =\alpha_{j+1} y_{j+1}(m+1)+y_{j}(m), \quad j \geq 1 .
\end{aligned}
$$

Choose $U_{j}=\alpha_{j} I$ and $c_{j}=y_{j}(m)$ for each $j \geq 1$ in Theorem 2.1. Then

$$
T(m)=y_{1}(m) I+\sum_{j=1}^{\infty} y_{j+1}(m)\left(A-\alpha_{j} I\right) \cdots\left(A-\alpha_{1} I\right) .
$$

Take $A \in B(X)$. Define

$$
L(x(m))=x(m+1) .
$$

To verify (2.4), we see that

$$
L\left(\sum_{j=0}^{\infty} y_{j+1}(m) T_{j} x_{0}\right)=\sum_{j=0}^{\infty} y_{j+1}(m+1) T_{j} x_{0}=\sum_{j=0}^{\infty} L\left(y_{j+1}(m) T_{j} x_{0}\right) .
$$

Finally, to check (2.5), we calculate

$$
\begin{aligned}
& L\left(c_{1} x_{0}\right)=c_{1} U_{1} x_{0}=\left[y_{1}(m+1)-\alpha_{1} y_{1}(m)\right] x_{0}=0, \\
& L\left(c_{j+1} T_{j} x_{0}\right)-c_{j+1} U_{j+1} T_{j} x_{0}-c_{j} T_{j} x_{0} \\
& =\left[y_{j+1}(m+1)-\alpha_{j+1} y_{j+1}(m)-y_{j}(m)\right] T_{j} x_{0}=0, \quad j \geq 1 .
\end{aligned}
$$

Note that

$$
\sup _{j \geq 1}\left\|U_{j}\right\|=\sup _{j \geq 1}\left\|\alpha_{j} I\right\|=\sup _{j \geq 1}\left|\alpha_{j}\right| \leq r
$$

hence Corollary 2.3 (i) holds. Similar to Lemma 3.2, we claim that

$$
\left|y_{j+1}(m)\right| \leq \frac{R^{m}}{(R-r)^{j}}, \quad j \geq 0 .
$$


It would follow that $y_{j+1}(m)=O\left((R-r)^{-j}\right)$ as $j \rightarrow \infty$ and Corollary 2.3 (ii) would be valid. Eq. (3.6) gives

$$
\left|y_{1}(m)\right|=\left|\alpha_{1}\right|^{m} \leq r^{m} \leq \frac{R^{m}}{(R-r)^{0}} .
$$

Suppose that (3.7) is true for $j$. Then from (3.6) we see that

$$
\left|y_{j+2}(m)\right| \leq \sum_{k=0}^{m-1}\left|\alpha_{j+2}\right|^{m-1-k}\left|y_{j+1}(k)\right| \leq \sum_{k=0}^{m-1} r^{m-1-k} \frac{R^{k}}{(R-r)^{j}} .
$$

But

$$
\frac{r^{m-1}}{(R-r)^{j}} \sum_{k=0}^{m-1}\left(\frac{R}{r}\right)^{k}=\frac{R^{m}-r^{m}}{(R-r)^{j+1}} \leq \frac{R^{m}}{(R-r)^{j+1}},
$$

which proves the claim since

$$
\left|y_{j+2}(m)\right| \leq \frac{R^{m}}{(R-r)^{j+1}} .
$$

As both conditions (i) and (ii) of Corollary 2.3 hold, (2.2) is satisfied and a solution of $x(m+1)=A x(m)$ is given by

$$
\begin{aligned}
x(m) & =T(m) x_{0}, \\
T(m) & =y_{1}(m) I+\sum_{j=1}^{\infty} y_{j+1}(m)\left(A-\alpha_{j} I\right) \cdots\left(A-\alpha_{1} I\right) .
\end{aligned}
$$

Since $R$ can be taken arbitrarily large provided $R>\|A\|+2 r$, (3.8) is valid for any sequence $\left(\alpha_{j}\right)_{j=1}^{\infty}$ in $\mathbb{C}$ such that $\left|\alpha_{j}\right| \leq r$ for $j \geq 1$. For instance, if we choose the constant sequence $\left(\alpha_{j}\right)_{j=1}^{\infty}=(\alpha, \alpha, \ldots)$ for some $\alpha \in \mathbb{C} \backslash\{0\}$, then (3.6) gives

$$
y_{1}(m)=\alpha^{m}, \quad y_{j+1}(m)=\frac{\alpha^{m-j}}{j !} \prod_{k=0}^{j-1}(m-k), \quad j \geq 1 .
$$

The latter is true because

$$
\begin{gathered}
y_{j+1}(m+1)-\alpha_{j+1} y_{j+1}(m)-y_{j}(m)=\frac{\alpha^{m+1-j}}{j !} \prod_{k=0}^{j-1}(m+1-k) \\
-\frac{\alpha^{m-j+1}}{j !} \prod_{k=0}^{j-1}(m-k)-\frac{\alpha^{m-j+1}}{(j-1) !} \prod_{k=0}^{j-2}(m-k)
\end{gathered}
$$




$$
=\frac{\alpha^{m+1-j}}{j !}\left[\prod_{k=0}^{j-1}(m-k+1)-(m+1) \prod_{k=0}^{j-2}(m-k)\right]=0 .
$$

Therefore,

$$
\begin{aligned}
T(m)= & \alpha^{m} I+\sum_{j=1}^{\infty}\left[\frac{\alpha^{m-j}}{j !} \prod_{k=0}^{j-1}(m-k)\right](A-\alpha I)^{j} \\
& =\alpha^{m} I+\sum_{j=1}^{m}\left[\frac{\alpha^{m-j}}{j !} \prod_{k=0}^{j-1}(m-k)\right](A-\alpha I)^{j} \\
& =\sum_{j=0}^{m}\left(\begin{array}{c}
m \\
j
\end{array}\right)(\alpha I)^{m-j}(A-\alpha I)^{j}=A^{m}
\end{aligned}
$$

and we recover the usual operator power. If we consider $x(m+1)=A x(m)$ with the initial condition $x(0)=x_{0}$, then $x(m)=A^{m} x_{0}$ is a solution. Taking any other uniformly bounded sequence $\left(\alpha_{j}\right)_{j=1}^{\infty}$ in $\mathbb{C}$, we see from (3.6) and (3.8) that $x(0)=T(0) x_{0}=x_{0}$. Assuming that the solution of the IVP is unique, we deduce that

$$
A^{m}=y_{1}(m) I+\sum_{j=1}^{\infty} y_{j+1}(m)\left(A-\alpha_{j} I\right) \cdots\left(A-\alpha_{1} I\right) .
$$

Similar to (3.4), (3.9) provides an infinite number of ways of representing the operator power.

Example 3.10 (Elaydi-Harris method for the matrix power). Continuing with the previous example, we now consider whether the sequence $\left(\alpha_{j}\right)_{j=1}^{\infty}$ can be chosen in such a way that the series (3.9) collapses to a finite sum. This is possible if $X$ is finite dimensional, say $X=\mathbb{R}^{n}$, due to the Cayley-Hamilton Theorem.

Let $x(m), x_{0} \in \mathbb{R}^{n}$. Suppose that $A \in M_{n}(\mathbb{R})$ is invertible and has eigenvalues $\lambda_{1}, \lambda_{2}, \ldots, \lambda_{n}$. Note that each eigenvalue is nonzero. Then $x(m+1)=$ $A x(m)$ simplifies to a system of $n$ linear first-order difference equations. Choose $\alpha_{j}=\lambda_{j}$ for $1 \leq j \leq n$ and $\alpha_{j}=0$ for $j \geq n+1$. The Cayley-Hamilton Theorem implies that

$$
\left(A-\alpha_{j} I\right) \cdots\left(A-\alpha_{1} I\right)=O, \quad j \geq n,
$$

where $O$ is the $n \times n$ zero matrix. Hence we have $x(m)=T(m) x_{0}$, where

$$
T(m)=y_{1}(m) I+\sum_{j=1}^{n-1} y_{j+1}(m)\left(A-\lambda_{j} I\right) \cdots\left(A-\lambda_{1} I\right)
$$


and

$$
y_{1}(m)=\lambda_{1}^{m} \quad y_{j+1}(m)=\sum_{k=0}^{m-1} \lambda_{j+1}^{m-1-k} y_{j}(k), \quad 1 \leq j \leq n-1 .
$$

If we impose the initial condition $x(0)=x_{0}$, then uniqueness of the solution yields

$$
A^{m}=y_{1}(m) I+\sum_{j=1}^{n-1} y_{j+1}(m)\left(A-\lambda_{j} I\right) \cdots\left(A-\lambda_{1} I\right)
$$

and we recover the Elaydi-Harris method for computing the matrix power [5].

Example 3.11 (Linear eigenvalue problem). Let $\lambda \in \mathbb{C}, A \in B(X)$ and $L x=\lambda x$. Hence we have the linear eigenvalue problem

$$
A x=\lambda x .
$$

Take $U_{j}=\alpha_{j} I$ for all $j \geq 1$, where $\left(\alpha_{j}\right)_{j=1}^{\infty}$ is some sequence in $\mathbb{C}$ to be determined. More specifically, let $r>0$ and $R>\|A\|+2 r$; hence $R>2 r>r$. Suppose that

$$
\alpha_{1}=\lambda, \quad \alpha_{j} \in D_{R-r}(\lambda)^{c} \cap \bar{D}_{r}(0), \quad j \geq 2 .
$$

Then

$$
\alpha_{1}=\lambda, \quad\left|\lambda-\alpha_{j}\right| \geq R-r, \quad\left|\alpha_{j}\right| \leq r, \quad j \geq 2 .
$$

The second inequality in (3.10) verifies Corollary 2.3 (i). For the sequence $\left(c_{j}\right)_{j=1}^{\infty}$, take

$$
c_{1}=1, \quad c_{j+1}=\frac{c_{j}}{\lambda-\alpha_{j+1}}, \quad j \geq 1
$$

Note that $c_{j+1}$ exists since $\left|\lambda-\alpha_{j+1}\right|>0$ for all $j \geq 1$. Thus

$$
c_{j+1}=\frac{1}{\prod_{k=2}^{j+1}\left(\lambda-\alpha_{k}\right)}, \quad j \geq 1
$$

and therefore from (3.10) we observe that

$$
\left|c_{j+1}\right| \leq \frac{1}{(R-r)^{j}}, \quad j \geq 1 .
$$

This verifies condition (ii) of Corollary 2.3. 
We see that

$$
\begin{aligned}
L\left(\sum_{j=0}^{\infty} c_{j+1} T_{j} x_{0}\right) & =\lambda \sum_{j=0}^{\infty} c_{j+1} T_{j} x_{0}=\sum_{j=0}^{\infty} \lambda c_{j+1} T_{j} x_{0} \\
& =\sum_{j=0}^{\infty} L\left(c_{j+1} T_{j} x_{0}\right) .
\end{aligned}
$$

Moreover,

$$
\begin{aligned}
& L\left(c_{1} x_{0}\right)-c_{1} U_{1} x_{0}=\left(\lambda-\alpha_{1}\right) x_{0}=0, \\
& L\left(c_{j+1} T_{j} x_{0}\right)-c_{j+1} U_{j+1} T_{j} x_{0}-c_{j} T_{j} x_{0} \\
& =\left(\lambda c_{j+1}-\alpha_{j+1} c_{j+1}-c_{j}\right) T_{j} x_{0}=0, \quad j \geq 1 .
\end{aligned}
$$

Hence $L$ satisfies (2.4) and (2.5). Therefore, for every $x_{0} \in X$, a solution of $A x=\lambda x$ is

$$
x=x_{0}+\sum_{j=1}^{\infty} \frac{1}{\prod_{k=2}^{j+1}\left(\lambda-\alpha_{k}\right)}\left(A-\alpha_{j} I\right) \cdots\left(A-\alpha_{2} I\right)(A-\lambda I) x_{0} .
$$

A special case is if $\lambda$ is an eigenvalue of $A$ with eigenvector $x_{0}$. Then the series on the right-hand side drops out and we get $x=x_{0}$.

For example, choose $r>0$ and $R>0$ such that $R-r>\|A\|+r$. Suppose that $|\lambda| \geq R-r$. Take $\alpha_{1}=\lambda$ and $\alpha_{j}=0$ for all $j \geq 2$. Then (3.10) is satisfied. We see that $\prod_{k=2}^{j+1}\left(\lambda-\alpha_{k}\right)=\prod_{k=2}^{j+1} \lambda=\lambda^{j}$ and a solution of $A x=\lambda x$ is

$$
x=x_{0}+\sum_{j=1}^{\infty} \frac{1}{\lambda^{j}} A^{j-1}(A-\lambda I) x_{0}, \quad x_{0} \in X .
$$

Example 3.12 (Fredholm integral equation). Consider the Sturm-Liouville system

$$
-x^{\prime \prime}(t)=\mu f(t) x(t)=0, \quad x(0)=x(1)=0 .
$$

Using Green's functions, we can rewrite this as the Fredholm integral equation

$$
x(t)=\mu \int_{0}^{1} g(t, u) f(u) x(u) \mathrm{d} u, \quad 0 \leq t \leq 1,
$$

where

$$
g(t, u)= \begin{cases}t(1-u) & \text { if } t \leq u \\ u(1-t) & \text { if } u \leq t\end{cases}
$$


It is known [6] that a nontrivial solution in $X=L^{2}[0,1]$ exists if

$$
|\mu| \geq\left[\int_{0}^{1} \int_{0}^{1} g(t, u)^{2} f(u)^{2} \mathrm{~d} u \mathrm{~d} t\right]^{-\frac{1}{2}} .
$$

Letting $\lambda=\frac{1}{\mu},(3.12)$ can be expressed as the operator equation

$$
\lambda x(t)=(A x)(t)=\int_{0}^{1} K(t, u) x(u) d u, \quad 0 \leq t \leq 1,
$$

where the kernel is $K(t, u)=g(t, u) f(u)$. For simplicity, take $x_{0}(t)=1$ for all $0 \leq t \leq 1$ without loss of generality. Hence

$$
\begin{gathered}
\left(A x_{0}\right)(t)=\int_{0}^{1} K(t, u) \mathrm{d} u=\int_{0}^{1} g(t, u) f(u) \mathrm{d} u, \\
y(t ; \lambda)=\left((A-\lambda I) x_{0}\right)(t)=\int_{0}^{1} g(t, u) f(u) \mathrm{d} u-\lambda .
\end{gathered}
$$

Note that

$$
\|A x\|^{2}=\int_{0}^{1}(A x)(t)^{2} \mathrm{~d} t=\int_{0}^{1}\left[\int_{0}^{1} K(t, u) x(u) \mathrm{d} u\right]^{2} \mathrm{~d} t .
$$

The Cauchy-Schwarz inequality yields

$$
\begin{aligned}
\|A x\|^{2} & \leq \int_{0}^{1}\left[\int_{0}^{1} K(t, u)^{2} \mathrm{~d} u\right]\left[\int_{0}^{1} x(u)^{2} \mathrm{~d} u\right] \mathrm{d} t \\
& =\|x\|^{2} \int_{0}^{1} \int_{0}^{1} K(t, u)^{2} \mathrm{~d} u \mathrm{~d} t
\end{aligned}
$$

or

$$
\|A x\| \leq\left[\int_{0}^{1} \int_{0}^{1} g(t, u)^{2} f(u)^{2} \mathrm{~d} u \mathrm{~d} t\right]^{\frac{1}{2}}\|x\| .
$$


This gives

$$
\|A\| \leq\left[\int_{0}^{1} \int_{0}^{1} K(t, u)^{2} \mathrm{~d} u \mathrm{~d} t\right]^{\frac{1}{2}}=\left[\int_{0}^{1} \int_{0}^{1} g(t, u)^{2} f(u)^{2} \mathrm{~d} u \mathrm{~d} t\right]^{\frac{1}{2}} .
$$

Following the previous example, take $r>0$ and $R>0$ such that

$$
\left[\int_{0}^{1} \int_{0}^{1} g(t, u)^{2} f(u)^{2} \mathrm{~d} u \mathrm{~d} t\right]^{\frac{1}{2}}<R-2 r .
$$

This would imply that $\|A\|<R-2 r$ or $R-r>\|A\|+r$. If

$$
\left[\int_{0}^{1} \int_{0}^{1} g(t, u)^{2} f(u)^{2} \mathrm{~d} u \mathrm{~d} t\right]^{-\frac{1}{2}} \leq|\mu|=\frac{1}{|\lambda|} \leq \frac{1}{R-r},
$$

then using (3.11), an explicit solution of (3.12) is

$$
x(t)=1+\sum_{j=1}^{\infty} \mu^{j}\left(A^{j-1} y\right)\left(t ; \frac{1}{\mu}\right), \quad y\left(t ; \frac{1}{\lambda}\right)=\int_{0}^{1} g(t, u) f(u) \mathrm{d} u-\frac{1}{\mu},
$$

provided $\mu$ satisfies (3.13).

\section{Discussion and concluding remarks}

In this article, we generalised the fundamental matrix and gave a new method to obtain an explicit solution for a class of linear operator equations that encompasses systems of ODEs, difference equations and fractional ODEs. This class also includes operator exponentials and operator powers, as well as eigenvalue problems and Fredholm integral equations. In the case when the underlying Banach space is $\mathbb{R}^{n}$, we recovered known methods for calculating the matrix exponential [10], matrix power [5] and fractional matrix exponentials [11]. The explicit solution is in the form of a series. We also gave sufficient conditions for the series to converge, as well as a result that provides the number of terms in the series to take to get to within a given accuracy.

For linear eigenvalue problems with $\lambda=1$, solving the operator equation $\lambda x=L x=A x$ is equivalent to finding a fixed point of the bounded 
linear operator $A$, i.e. finding $x \in X$ such that $A x=x$. One classical way of doing this is via the use of the Contraction Mapping Theorem [15]. The method we propose here is an alternative approach to estimating the fixed point that does not rely on contractions and also provides error bounds.

We can also study the nonhomogeneous problem

$$
L x=A x+y,
$$

where $y \in X$ is given and $L$ is an invertible bounded linear operator such that $\|A\| \leq \frac{1}{\left\|L^{-1}\right\|}$. Therefore $L-A$ is invertible and

$$
\left\|(L-A)^{-1}\right\| \leq \frac{\left\|L^{-1}\right\|}{1-\left\|L^{-1}\right\|\|A\|}
$$

(see [6, p. 240], for instance). We claim that for any $x_{0} \in X$, an explicit solution of $L x=A x+y$ is

$$
x=T x_{0}+(L-A)^{-1} y .
$$

But since $T x_{0}$ is a solution of the homogeneous equation $L x=A x$, this follows easily from

$$
L x-A x=(L-A)\left(T x_{0}\right)+(L-A)(L-A)^{-1} y=y .
$$

In their seminal work, Moler and Van Loan [8] considered different ways to compute the matrix exponential $\mathrm{e}^{t A}$. Most of the methods rely on being able to compute the eigenvalues and eigenvectors of the matrix $A$, which is a highly nontrivial issue especially when there are repeated eigenvalues. The method we propose here relies instead on choosing a sequence $\left(\alpha_{j}\right)_{j=1}^{\infty}$ in $\mathbb{C}$ that satisfies the (relatively mild) conditions of Corollary 2.3. Then Corollary 2.5 gives the number of terms to take in the series for $\mathrm{e}^{t A}$ to get a desired accuracy, while avoiding the calculation of the eigenvalues of $A$.

A natural question to ask is whether the method proposed here will also work when $A$ is an unbounded linear operator, e.g. a derivative operator. There are two important considerations that will have to be addressed. The first one is that the domains for the operators in the sequences of Theorem 2.1 should all be the same. The other, more important, issue is the question of convergence of the series in (2.6). We will have to choose a sequence $\left(U_{j}\right)_{j=1}^{\infty}$ that would somehow annihilate the operator appearing inside the series, thus bypassing the problem of convergence. Alternatively, we could consider an infinite dimensional version of the Cayley-Hamilton Theorem along the lines of [14], although this would only hold for a restricted class of unbounded operators. The extension to unbounded operators is currently work in progress. 


\section{References}

[1] F. Brauer, C. Castillo-Chávez, Mathematical Models in Population Biology and Epidemiology, Springer, New York (2012).

[2] F. Brauer, J.A. Nohel, The Qualitative Theory of Ordinary Differential Equations, Dover, New York (1989).

[3] G. de Vries, T. Hillen, M. Lewis, J. Müller, B. Schöfisch, A Course in Mathematical Biology: Quantitative Modeling with Mathematical and Computational Methods, SIAM, Philadelphia (2006).

[4] S.N. Elaydi, An Introduction to Difference Equations, Springer, New York (2000).

[5] S.N. Elaydi, W.H. Harris, Jr., On the computation of $A^{N}, S I A M$ Rev., 40, No 4 (1998), 965-971.

[6] D.H. Griffel, Applied Functional Analysis, Wiley \& Sons, New York (1981).

[7] K.S. Miller, B. Ross, An Introduction to the Fractional Calculus and Fractional Differential Equations, Wiley, New York (1993).

[8] C. Moler, C. Van Loan, Nineteen dubious ways to compute the exponential of a matrix, twenty-five years later, SIAM Rev., 45, No 1 (2003), 3-49.

[9] I. Podlubny, Fractional Differential Equations, Mathematics in Science and Engineering 198, Academic Press, San Diego (1999).

[10] E.J. Putzer, Avoiding the Jordan canonical form in the discussion of linear systems with constant coefficients, Amer. Math. Monthly, 73, No 1 (1966), 2-7.

[11] M.R. Rodrigo, On fractional matrix exponentials and their explicit calculation, J. Differential Equations, 261, No 7 (2016), 4223-4243.

[12] H. Schäfer, E. Sternin, R. Stannarius, M. Arndt, F. Kremer (1996), Novel approach to the analysis of broadband dielectric spectra, Phys. Rev. Lett., 76, No 12 (1996), 2177-2180.

[13] D. Slepian, Some comments on Fourier analysis, uncertainty and modeling, SIAM Rev., 25 No 3 (1983), 379-393. 
[14] A.M. Teguia, Extensions of the Cayley-Hamilton Theorem with applications to elliptic operators and frames, Electronic Theses and Dissertations, Paper 1024 (2005), http://dc.etsu.edu/etd/1024.

[15] E. Zeidler, Applied Functional Analysis: Applications to Mathematical Physics, Applied Mathematical Sciences Series, Vol. 108, Springer-Verlag, New York (1995). 